Beograd

\title{
UNE DIFFÉRENCE FONDAMENTALE DANS LA LANGUE BIBLIQUE ENTRE LE ROUMAIN ET L'AROUMAIN
}

Sans nous ingérer au problème si l'aroumain présente un dialecte historique de la langue roumaine ou s'il s'agit ici d' une langue à part ${ }^{1}$, nous voulons maintenant mettre en évidence une différence entre les deux idiomes à la base de l'expression que les deux langues ou dialectes appliquent aujourd'hui dans le style biblique.

Il est vrai que la linguistique traditionnelle regarde les parlers roumains balkaniques, c'est-à-dire l'aroumain, le mégléno-roumain et l'istro-roumain comme des dialectes historiques du roumain, qui représenterait à son tour, un dialecte historique, c'est-à-dire le daco-roumain, devenu langue officielle et nationale de la Roumanie qui dispose d'une littérature populaire et artistique pluriséculaire. ${ }^{2}$ Les autres dialectes n'avaient pas de conditions historiques indispensables pour se lever au niveau du roumain, à savoir pour devenir langues littéraires et nationales parce que la plupart des sujets parlant ces dialectes étaient bilingues et sans création littéraire excepté la création populaire.

Naturellement, il est impossible de parler de la conscience nationale des personnes parlant ces dialectes en famille. Tandis que l'istro-roumain et le mégléno-roumain sont réduits aujourd'hui à un nombre insignifiant des sujets parlant (le nombre des istro-roumains ne dépasse pas mille personnes et celui des mégléno-roumains cinq mille et demi), ${ }^{3}$ le nombre des personnes parlant

1. Cfr. M.D. Savic, L'aroumain entre dialecte et langue (en Linguistica XXVII, Ljubljana 1987, pp. 63-72). Il s'agit d'une conférence tenue le 6 mai 1987 à l'Université de Freiburg. Les idées que nous avons exposées en cette occasion sont en partie originales et en partie liées aux théories dont les auteurs sont des linguistes roumains. Cfr. George Ivănescu, Istoria limbii romane, lasi 1980, pp. 30-82, passim, à savoir les chapitres III et IV, dont les titres sont Dialectele limbii románe în epoca moderna et Teritoriul de formatie a limbii romane si a poporului român. Le livre de G. Ivănescu a eu notre présentation très favorable, surtout dans les pages concernant l'époque ancienne de la langue roumaine (voir Balcanica XII, Beograd 1981, pp. 182-185).

2 Une contribution à cet égard a été offerte aussi pour le linguiste yougoslave Radu Flora, Rumunski banatski govori u svetlosti lingvisticke geografije, Beograd 1969, passim.

3 Cfr. Tratat de dialectologie romaneased, Craiova 1984, coordonné de Valeriu Rusu: aroumain, pp. 423-475 (Nicolae Saramandu), mégléno-roumain, pp. 476-549 (Petar Atanasov), istro-roumain, pp. 550-590 (August Kovaðec). 
Paroumain s'élève à un million et deux cent mille selon les données statistiques offertes par les organisations culturelles des Aroumains vivant en Europe (en premier lieu en Allemagne et en France) et aux États-Unis d' Amérique). ${ }^{4}$

Avant de passer au problème qui nous intéresse ici, nous avons la tâche d'expliquer mieux qui sont les Aroumains (connus dans les Balkans sous le nom de "Tsintsar", peut-être grâce au phonème $t s$, qui est très fréquent dans leur idiome). In est bien connu aussi qu'ils ont donné leur grande contribution à la formation de tous les peuples balkaniques, ce qui a fait apparaître la sentence: Si les peuples balkaniques peuvent être présentés comme des pains, en ce cas les Aroumains présentent le levain qui a rendu possible la cuisson de ces pains.

Sans négliger la première mention d'un idiome néolatin balkanique "torna, torna frate" de l'an 587, régistrée chez un auteur byzantin et liee aujourd'hui à l'aroumain par les linguistes modernes ${ }^{5}$, nous pouvons constater la première mention de la population parlant cet idiome au $\mathrm{X}^{\mathrm{e}}$ siècle sur un territoire faisant partie de Byzance pour continuer sa vie de masse compacte, après la chute de Byzance, sur le même territoire sous la domination ottomane. Il s'agit de la Valaquie (en Grèce d'aujourd'hui), citee ainsi dans les documents historiques. Mais les evénements historiques n' etaient jamais favorables aux Aroumains, ce qui se voit spécialement dans notre siècle. Si le $\mathrm{XX}^{\mathrm{e}}$ siècle a rendu possible la renaissance des états nationaux européens et surtout de ceux dans les Balkans, il n'a rien offert à ce petit peuple dont l'idiome nous intéresse. La retraite de l'Empire Ottoman qui fut contraint à céder toute la région peuplée par les Aroumains aux états balkaniques était très défavorable à la vie et au développement ultérieur de ce petit groupe linguistique et ethnique. Répartis en quatre états, après les guerres balkaniques (Grèce; Albanie, Yougoslavie, Bulgarie) et, après la première guerre mondiale, transférés aussi en Roumanie, les Aroumains ne sont plus compacts comme ils l' étaient au temps de l'Empire Ottoman où ils avaient eu des privilèges concernant le commerce et la vie culturelle et ethnique. Mais s'ils avaient été en possibilité de préserver leur langue, leurs coutumes et leur substance ethnique, ils avaient été soumis à l'influence roumaine grâce au privilège qu'avait obtenu la Roumanie dans la deuxième moitié du siècle passé de réaliser l'instruction scolaire en langue roumaine dans les régions de l'Empire Ottoman où vivaient les Aroumains. Autrement dit, il s'agissait d'une autonomie culturelle et linguistique

4 Ces données statistiques ont été répétées plusieurs fois aussi pendant les deux congrès internationaux des Aroumains qui ont eu lieu en 1985 à Mannheim et en 1988 à Freiburg.

5 Cfr. Valeriu Rusu, "Toma, torna fratre" dans la perspective de l'ethnographie balkanique (en Mélanges de linguistique dédiés à la mémoire de Petar Skok, Zagreb 1985, pp. 437-439). 
qui était très utile à cette population; mais d'autre côté, les écoles fonctionnaient en langue roumaine et non pas en aroumain. ${ }^{6}$

Cet idiome (dont nous avons le premier document écrit au XVII ${ }^{\mathrm{e}}$ siècle) avait déjà, à la différence de l' istro-roumain et du mégléno-roumain, une tradition culturelle qui était mise en premier lieu à Moscopoïe (ou Voscopoïe), une ville aroumaine, qui se trouvait en Albanie du Sud et qui avait plus de 50.000 habitants en disposant aussi des imprimeries qui publiaient les livres en aroumain. Cependant, cette ville avait été détruite par Ali-pacha de Janina et ses habitants avaient été contraints dè quitter leur foyer.

Si l'on peut parler d'une conscience nationale ou au moins linguistique des Aroumains, on doit constater qu'elle est due aux Aroumains qui avaient vécu à Vienne et en Autriche dans la première moitié du siècle passé en faisant connaissance ainsi que les autres peuples vivant en Autriche et dans les Balkans, des idées proclamées par la Révolution française et par le romantisme. Mais les idées nouvelles pénétraient à pas lent dans les Balkans, en particulier dans le Midi. ${ }^{7}$

Il nous semble que les privilèges que les Aroumains pouvaient profiter des écoles en langue roumaine avaient été très inconvenables en ce qui concerne l'autonomie è la spécialité de ce groupe ethnique et linguistique. Il faut souligner que cette population, soit-elle originaire du territoire où elle se trouve aujourd'hui pour des siècles sans arrêt ou soit arrivée ici de la Mésie Supérieure selon l'avis de quelques historiens et linguistes ${ }^{8}$ (nous refusons toute idée représentant les

6 Cfr. Max Demeter Peyfuss, Die Arumunische Frage, ihre Entwicklung von den Ursprüngen bis zum Frieden von Bucarest und die Haltung Österreich-Ungarns, Wien 1974, passim. Nous renvoyons à notre presentation favorable de ce livre (en Zbornik za istoriju Matice stpske 14, Novi Sad 1976, pp. 219-222).

7 Ibidem.

8 D'après une théorie, la population aroumaine descendue du Nord des Balkans, était d'origine illyrienne ou thrace ou de toutes les deux. Une autre théorie la considère comme héritière des anciennes populations balkaniques preromanes (aussi bien des tribus illyriennes et thraces qui vivaient au Nord de la Grèce), qui y sont autoctones. Il est impossible d' accepter l'affirmation offerte dans le livre d'Achille Lazarou L'aroumain et ses rapports avec le grec, Thessaloniki 1986, qui considère que les Aroumains sont des Grecs romanisés et qui insiste à la grécité primordiale de cette population. En terminant son exposé, l'auteur écrit littéralement: "Ce vocabulaire riche et varié (il s'agit du lexique d'origine grecque en aroumain) qui recouvre toutes les situations de la vie quotidienne montre clairement que les Aroumains sont des Grecs qui malgré le fait que l'aroumain leur fut imposé comme seconde langue, préservent une partie du trésor inestimable de la première langue." (p. 259) Mais, à notre avis, ce n'est pas prouve. Ajoutons que toutes affirmations purement linguistiques de cet auteur (qui est d'origine aroumaine) sont acceptables, mais ses affirmations restent peu croyables ou discutables quand on passe aux problèmes historiques. Cfr. notre critique de ce livre (Linguistica XXVII, Ljubljana 1987, pp. 189-191). 
Aroumains comme héritiers des anciens Macédoniens d'Alexandre le Grand, idée qui fut mise en relief plusieurs fois au Congrès des Aroumains et dans leur presse comme sans fondement ${ }^{9}$ ), diffère beaucoup de celle de langue roumaine qui se trouve à gauche du Danube parce que les deux populations avaient été séparées l'une de l'autre pendant à peu près mille ans si nous tenons compte des grandes invasions des peuples à partir du premier millénaire de notre ère, en particulier de l'arrivée des Slaves méridionaux et, plus tard, des Hongrois qui ont coupé toutes les relations entre le monde roman du Nord du Danube et celui du Sud. ${ }^{10}$

En abandonnant la préhistoire se référant au problème de l'origine de la petite ethnie dont nous parlons, il faut dire que les deux congrès des Aroumains tenus jusqu'aujourd'hui (un a eu lieu à Mannheim en 1985 et l'autre à Fribourg en 1988) se sont occupés des questions actuelles concernant l'intention de faire continuer à vivre le groupe aroumain en préservant ses spécialités ethniques, folkloriques et linguistiques sans s'ingérer au problème politique. Au cours des travaux des dits congrès, les représentants des Aroumains à l'aide des autres participants, parmi lesquels étaient aussi des linguistes, ont réussi à fixer leur alphabet qui diffère sensiblement de l'alphabet roumain déclarant en même temps que l'aroumain represente une langue à part. Il s'agissait soit du problème de l'alphabet soit de celui concernant la langue. Quant à l'alphabet, il faut souligner que le moment décisif qui a rendu possible sa réalisation actuelle, c'était la présence des Aroumains vịvant en Angleterre ou aux États-Unis d'Amérique dont la langue officielle est l'anglais qui possède l'alphabet sans signes diacritiques. Les Aroumains anglo-américains quoiqu' ils n'aient pas été en majorité aux deux congrès ont réussi à imposer leur proposition, c'est-à-dire l'acceptation du système alphabétique existant en anglais. Cette décision a été adoptée sans doute grâce aux motifs techniques, car les machines à écrire et les imprimeries ne disposent pas toujours de signes diacritiques, ce qui complique la communication écrite en aroumain. En adoptant le nouvel alphabet, les roumains, à la différence de ces signes qui sont indispensables en roumain (par ex. $s$ et $t)^{11}$, les ont éliminés n'en préservant qu' $\bar{a}$, ce qui - à notre avis - ne suffit pas, parce que le dernier signe ne marque pas la différence entre les voyelles $\breve{a}$ et $\hat{\imath}$ existant ainsi en roumain qu'en aroumain. Naturellement, la reforme de l'alphabet exige une période plus longue pour pouvoir être réalisée définitivement, ce que montreront aussi les textes que nous -allons citer tout de

9 Il s'agit - ̀̀ notre avis - d'une disposition romantique qui présente un phénomène normal et présent dans tous les groupes ethniques sans tradition écrite insistant à découvrir leur origine.

10 Cr. M. D. Savic, La conjugaison aroumaine en relation aux conjugaisons des autres langues balkaniques (en Balcanica XX, 1989, pp. 341-346). Il s'agit de la communication présentée au deuxième Congrès international des Aroumains tenu en 1988 à Freiburg. Nous y avons souligné en premier lieu les divergences existant entre le roumain et l' aroumain.

11 Les deux graphèmes $s$ et $t$ sont remplacés par les graphèmes.sh et $t s$ en aroumain. 
suite. Le nouvel alphabet est appliqué aujourd'hui dans toutes les publications aroumaines qui apparaissent en Europe et en Amérique, par ex., "Zborlu a nostru" (Notre parole), une revue publiée à Freiburg (Allemagne).

Sans doute, il jouait un rôle prépondérant à la systématisation de l'alphabet aroumain l'oeuvre de Mihail Boiagi, la première grammaire de langue aroumaine, publiée en 1813 a Vienne appliquant pour la première fois les caractères latins dans un idiome balkanique (ce qui a été fait plus tard par les Roumains dans la deuxième moitie du siècle dernier, par les Albanais en 1908 et les Turcs en 1927). Soulignons aussi que la dite grammaire exposée en même temps en grec et en allemand, et destinée en premier lieu aux Aroumains vivant en Autriche et en Turquie a été dénoncée et proscrite de la part du Patriarchat orthodoxe de Constantinople dont la langue officielle était le grec. En même temps, il faut rappeler que l'oeuvre de Boiagi présente la première grammaire d'une langue balkanique. ${ }^{12}$

Quant à la langue, nous ne mentionnons que le lexique qui diffère beaucoup entre le roumain et l'aroumain, ayant en vue que le premier connaît surtout l'influence slave, tandis que le deuxième, étant limitrophe avec le grec, en a subi une grande influence. ${ }^{13}$ Ajoutons encore que la communication linguistique entre les Roumains et les Aroumains n'est pas facile aujourd'hui se réduisant approximativement à la communication pareille à celle qui existe entre le russe et le serbocroate.

Les congrès ont mis en évidence qu'il y a des écrivains qui publient en aroumain, spécialement des poètes.

En retournant au problème qui se trouve au centre de notre intérêt, c'est-à-dire à la différence entre les moyens qui servent à exprimer le temps passé dans la langue biblique, il faut dire que nous avons effectué les recherches des textes. Notre intention de nous occuper du texte biblique en aroumain d'il y a quelques années, lorsque nous avons attiré l'attention à cette question en écrivant du même problème

12 L'oeuvre de Boiagi a vu encore trois réeditions, la première en 1857 et la deuxième en 1915 (toutes les deux à Bucarest). La dernière édition, publiée à Freiburg à l'occasion des $\mathbf{1 7 5}$ ans de l'apparition de cette oeuvre est due au professeur universitaire de Freiburg, Vasile Barba, dont l'intention était de renouveler ce livre qui n'a pas perdu son actualité malgré le temps passé.

A. Lazarou, op. cit, passim, surtout les pages concernant le lexique. 
dans les langues balkaniques ${ }^{14}$ et aussi dans un certain nombre de langues parlees en Europe ${ }^{15}$, n'a pas donné fruit parce que les personnages parlant l'aroumain et n'étant pas sans érudition linguistique nous ont proposé de ne pas profiter de la traduction aroumaine existante, car elle présente une roumainisation de l'aroumain. Ils nous ont annoncé en même temps l'apparition d'une nouvelle Bible qui serait conforme à toutes les particularités d'un aroumain traditionnel compréhensible et intime à tous les sujets parlant cet idiome.

Bien que nous ayons profité jusqu'aujourd'hui de nos recherches réalisées à base de l'Évangile selon saint Matthieu, nous avons à présent à notre disposition l'Évangile selon saint Marc car les autres parties du Nouveau et de l'Ancien Testament n'ont pas encore connu une traduction correcte (au sens d'une expression purement aroumaine). ${ }^{16}$ En nous arrêtant au deuxième chapitre de l'Evangile mentionné, nous pouvons constater dès le premier coup d'oeil une grande différence entre les textes roumains et aroumains concernant surtout le vocabulaire, les autres particularités n'étant pas moins évidentes surtout lorsqu'il s'agit des moyens morpho-syntaxiques à savoir aussi du problème qui nous intéresse. N'en donnant qu'un exemple, disons que le chapitre roumain en parole porte le sous-titre "Vindecarea slăbănogului" tandis que le sous-titre du texte aroumain est "Vindicarea a paraliticlui" ce qui nous montre l'influence de la langue grecque sur le texte aroumain. ${ }^{17}$ (Le sous-titre du même texte en traduction française est "Jésus guérit un paralytique" ce qui se rapproche de la traduction en aroumain et non de celle en roumain ce que nous allons voir mieux en continuant.) ${ }^{18}$ Il est vrai que cette différence est évidente dans beaucoup d' articles de ce chapitre ainsi que dans l'Évangile entier selon Marc que nous avons eu dans nos mains.

En quittant le problème du lexique qui sort du cadre de notre intérêt actuel, passons aux paradigmes verbaux qui servent à exprimer le passé dans le deuxième

14 Crr. M. D. Savic, Quelques interrelations des langues des Pays du sudest européen concernant le système verbal (en Zeitschrift für Balkanologie, Band XVI, Westberlin 1980, pp. 139-147), où le point de départ se réduit aux moyens synthétiques ou analytiques servant à exprimer le temps passé dans les textes bibliques. Il s'agit de la communication présentée au IV congrès de balkanologie qui a eu lieu Ankara en 1979.

Voir M. D. Savic, Stil i jezik Vukovog prevoda "Novog zavjeta" iz današnje perspektive (u okviru erropskih jezika) (en Sastanak slavista u Vukove dane 8, Beograd 1978, t. 1., pp. 141-145). Nous avons dépouillé l'édition aroumaine suivante: Sînta Evanghelie di la Marku, Editura Avdella (traduction de Apostol Caciuperi).

17 Pour le roumain nous nous sommes servis de: Biblia sau Sfinta scriptura a Vechiului si Noului testament, sine anno. Mais l'éditeur souligne qu'il s'agit d'une traduction nouvelle.

Nous offrons le texte français sur la base du Nouveau Testament illustré en français courant (traduit d' après le texte grec), Paris 1973, Alliance biblique universelle. 
chapitre des deux traductions analysees: tandis que la traduction roumaine montre 48 passés composés et 20 imparfaits sans aucun passé simple, la traduction aroumaine nous offre 33 passés simples, 18 imparfaits et seulement 5 passés composés, ce qui veut dire - si nous négligeons pour le moment l'imparfait réduit à peu près au même nombre dans les deux traductions - que la narration en roumain est effectuée par la forme composée du passé et que l'aroumain n'y connaît que la forme simple. Quant à la forme composée de l'aroumain, elle est réservée exclusivement au dialogue.

Illustrons notre affirmation par un exemple textuel où se trouvent toutes les trois formes verbales exprimant le temps passé tant en roumain qu'en aroumain!

Nous donnons d'abord le texte roumain:

1. După cîteva zile, Isus S'a întors în Capernaum. S’a auzit că este în casa,

2. și s-au adunat înđată aşa de mulți că nu putea să-i mai încapă locul dinaintea ușii. El le vestea Cuvîntul.

3. Au venit la el nişte oameni, cari l-au adus un slăbănog, purtat de patru insi.

4. Fiindcă nu putea să ajunga pînă la $\mathrm{El}$, din pricina norodului, au desfăcut acoperişul casei unde era Isus, şi, după ce l-au spart, au pogorît pe acolo patul, în care zăcea slăbănogul. iertate!"

5. Cînd le-a văzut Isus credința, a zis slăbănogului: "Fiule, păcatele îti sînt

6. Unii de cărturari, cari erau de față, se gîndeau în inimile lor:

7. "Cum vorbește omul acesta astfel? Huleste? Cine poate să ierte păcatele decît numai Dumnezeu?"

8. Îndată, Isus a cunoscut, prin đuhul Său, că ei gîndeau astfel în ei, si le-a zis: "Pentru ce aveţi astfel de gînduri în inimile voastre?

9. Ce este mai lesne: a zice slăbănogului: "Păcatele îti sînt iertate", ori a zice: "Scoală-te, ridică-ți patul, şi umblă?"

10. Dar, ca să stiti că Fiul omului are putere pe pămînt să ierte păcatele,

11. "Tie îţi poruncesc", a zis El slăbănogului - "scoală-te, ridica-ţi patul, si du-te acasă."

12. Si îndată, slăbănogul s'a sculat, şi a ridicat patul, şi a iesit afară în faţa tuturor; aşa că toti au rămas uimiţi, si slaveaù pe Dumnezeu, si ziceau: "Niciodata n’am văzut aşa ceva!"

Nous répétons maintenant la traduction aroumaine: ${ }^{19}$

19 Il faut remarquer que l'auteur applique l'alphabet roumain et non pas l'alphabet aroumain, vu que ce dernier est entré en vigueur plus tard. 
1 Iară după vîrna ndouă dzîle, iară intră Isus tu Capernaum,

2 S-cîndu si-avdzî că easte tu casă, si-adunară multi, că nu ma ncapea nitii pri ningră ușe; s-elu lă predica a loru zborlu.

3 Aṭumtea viniră la nîsu (nîste oamîni), aducîndului unu paraliticu, pri cari l-purtau patru insi.

4 Ma niputîndalui eli s-lủ aspună alu Isus di itia a mulţîmilei, dizvăliră citia ditu partea iu era, s-făcîndalui una disclidzătură, dipusiră patlu tu cari dzătea paraliticu.

5 Iara Isus, vidzîndalui pistea a loru, dzîse a paraliticlui: "Hiliu, ță si liartă amărtiile".

6 Și șideau aclo unili di cărturari, minduindalui tu iniñile a loru:

7 "Tră te zburaște aşi? Aestu blastima, cari poate s-liarta amărtiile nafoara di unu dumnidzău?"

8 S-trîoară cunuscîndului Isus cu duhlu a lui că aşi minduiau tu nîși, lă dzîse a loru: "Tră țe minduit aeste tu iniñile a voastre?

9 Te easte ma lișoru tră dzîcă a paraliticlui:. Tă și liartă amărtiile; sau s-dzîti: scoală-tii, lia-ţ patlu ş-imnă?

10 Ma tra si știł că hililu a omului are puteare tra s-jiartă amărtiile pr locu, (dzîse a paraliticlui):

11 Tă dzîcu: scoală-ti, lia-t patlu s-ñerdzi la casa a ta".

12 Ş-trîoară si sculă, s-muntînda-și patlu, s-duse di fạ̧a cu tuţ, că s-ciudiseau tưf s-măreau pri dumnidzău, dzîcîndului: "Vîrnă oară nu ma avemu vidzuta aşi tivă".

Avant de commencer à discuter sur la situation constatée dans les deux traductions, nous croyons utile de mettre à la disposition du lecteur une traduction française moderne du même texte en lui offrant en même temps la possibilité de suivre les deux textes, surtout de celui qui ne connaît pas assez les deux langues traitées.

Voici le texte français:

2 Quelques jours plus tard, Jésus revint à Capernaüm, et l'on apprit qu'il était à la maison. Une si grande foule s'assembla qu'il ne restait plus de place, pas même dehors devant la porte. Jésus leur donnait ses enseignements. Quelques hommes arrivèrent alors, lui amenant un paralytique porté par quatre d'entre eux. Mais ils ne pouvaient pas le présenter à Jésus, à cause de la foule. Ils ouvrirent alors le toit au-dessus de l'endroit où était Jésus; par le trou qu'ils avaient fait, ils descendirent le paralytique étendu sur sa natte. Quand Jésus vit la foi de ces hommes, il dit au paralytique: "Mon fils, tes péchés sont pardonnés." Quelques maîtres , qui étaient assis là, pensaient en eux-mêmes: "Comment cet homme ose-t-il parler contre Dieu? Qui peut pardonner les péchés? Dieu seul le peut!" Jésus sut aussitôt ce qu'ils 
pensaient et leur dit: "Pourquoi avez-vous de telles pensées? Est-il plus facile de dire au paralytique: 'Tes péchés sont pardonnés', ou de dire: 'Lève-toi, prends ta natte et marche?' Mais je veux que vous sachiez que le Fils de l'homme a le pouvoir sur la terre de pardonner les péchés."

Il adressa alors ces mots au paralytique: "Je te le dis, lève-toi, prends ta natte, et rentre chez toi!"

Aussitôt, tandis que tout le monde le regardait, l'homme se leva, prit sa natte et partit. Hs furent tous frappés d'étonnement; ils louaient Dieu et disaient: "Nous n'avons jamais rien vu de pareil!"

En comparant les trois textes cités, nous pouvons constater - comme nous l'avons déjà mentionné - que le texte aroumain correspond au texte français en ce qui concerne le problème dont nous discutons, tandis que le texte roumain nous fait voir ses propres particularités, à savoir la généralisation du passé composé qui assume à son tour outre le rôle du passé composé français ou aroumain, aussi celui du passé simple français ou aroumain. (Du point de vue purement stylistique qui dépasse le cadre de notre intérêt d'aujourd' hui, voire les formes du verbe servant à exprimer le temps passé, les textes roumain et aroumain sont plus conformes que ne le soit le texte français.)

Il est bien connu que la concurrence qui existe entre les formes simples et les formes composées se développe au détriment des premières; un procès opposé n'a pas été constaté jusqu'aujourd'hui. ${ }^{20}$ Il est normal que ce procès a embrassé un vaste territoire européen ne pas réussissant à atteindre la périphérie ce qui est rendu évident si nous examinons les moyens servant à exprimer le temps passé en portugais, en espagnol, en albanais et en grec moderne. ${ }^{21}$ Il s'agit des procès qui se sont accomplis dans un grand nombre de langues romanes et germaniques ce qui n'est pas toujours visible dans l'expression écrite ou littéraire qui a inconsciemment recours aux moyens archaïsants et dépassés. ${ }^{22}$ Il faut ajouter encore que les

20 Au sens de notre interêt, il faut mentionner une contribution importante offerte par Emilio Alarcos Llorach, Perfecto simple y perfecto compuesto (pp. 13-49), dans son livre Estudios de gramatica funcional del español, Madrid 1970 qui, s'occupant de ce problème en espagnol, le suit de siècle à siècle jusqu'à nos jours en appliquant les termes "presente gramatical" et "presente ampliado" quand il s'agit des deux fonctions differentes du passé composé.

21 Nous renvoyons aux données statistiques exposées dans notre contribution mentionné Stil $i$ jezik Vukovog prevoda "Novoga zavjeta"...

22 Cfr. Antoine Meillet, Sur la disparition des formes simples du préterit (en Linguistique historique et linguistique générale I, Paris 1926) qui décrit en forme succinte le problème en question dans le domaine indo-européen ouvrant les investigations et présentant en même temps la situation dans les langages de diverses langues. 
linguistes européens, en premier lieu les romanistes et les germanistes qui se sont occupés de ce problème, aient oublié le monde slave et leurs langues très importantes quand on insiste de résoudre le problème en question, puisque les langues slaves orientales et occidentales (excepté le sorabe qui continue à imiter la syntaxe allemande) ainsi que le slovène parmi les langues slaves méridionales, ont perdu les formes simples du passé. ${ }^{23} \mathrm{La}$ disparition des dites formes est évidente récemment aussi en serbocroate, où les deux dialectes occidentaux ne connaissent pas ces formes depuis le commencement du siècle passé tandis que le dialecte oriental présente - au sujet de ce problème - une phase de transition: nous n'y trouvons - en parlant d'une langue purement littéraire moderne - que le passé simple des verbes perfectifs sans aucun imparfait (le siècle passé nous offre aussi des passés simples des verbes imperfectifs). Le bulgare et le macédonien ont préservé à leur tour les formes simples du passé (c'est-à-dire l'aoriste et l'imparfait) conformément à un système syntaxique influencé par la langue turque qui prend en consideration qu'il s'agit d'une action passée dont le personnage qui parle est un témoin oculaire ou s'il s'agit d'une action que ce personnage transfère d'après le récit d'un autre personnage. Quant aux parlers albanais urbains, ils ne sont pas exemptés de cette influence turque bien que la langue albanaise en principe ait recours à la forme simple ainsi que le grec soit dans sa phase ancienne soit dans sa phase moderne.

Avant de conclure notre exposé, nous pouvons souligner que la différence entre le roumain et l'aroumain dans le domaine que nous avons examiné est due en premier lieu à la position géographique de chacune des deux langues ou dialectes dont la première, se trouvant très proche du centre de l'irradiation de l'innovation linguistique ne pouvait éviter le phénomène qui est évident dans toutes les langues de l'Europe Centrale-Orientale et Occidentale, tandis que l'autre, se trouvant à la périphérie européenne, n'a été que partiellement soumise à cette innovation. L'aroumain a été sans doute en ce qui concerne notre problème principalement sous l'influence de la langue grecque en préservant toutefois une phase linguistique archaïsante qui n'a pas changé dans le macédonien et le bulgare ce qui est évident dans plusieurs traductions de la Bible en serbocroate. ${ }^{24}$ D'autre côté, le roumain, bien qu'il fasse partie des langues balkaniques, se conforme ici aux langues slaves orientales et occidentales qui ont depuis longtemps perdu les formes simples pour

23 En essayant de résoudre les problèmes des langues romanes et germaniques, les linguistes occidentaux oublient de recourir aux langues slaves qui pourraient être très utiles. Au sujet de notre problème, citons une exception qui se réfère au passé composé en portugais: Horst G. Klein, Algumas observações sobre a categonização do sistema verbal português (en Boletim de filologia, tomo XXII, Lisboa 1973, pp. 295-301).

24 Voir les tableaux synoptiques concernant ce problème en Stil $i$ jezik Vukovog prevoda "Novog zavjeta"... 
exprimer le temps passé. ${ }^{25}$ Soulignons seulement que le passé simple n'est pas une forme vivante en Moldavie déjà à partir du siècle passé. ${ }^{26}$

Il n'est pas nécessaire de souligner que le texte biblique exige un style particulier qui doit être en même temps littéraire et compréhensible à un large public. Autrement dit, un texte pareil doit refleter la dignite religieuse non celle privée de sacralité et de misticisme. Naturellement, il fallait tout d'abord transférer littéralement tous les dogmes dont nous offre un bon témoignage la traduction en vieux slave effectuée d'après l'original grec et où l'on voit que le traducteur respecte beaucoup de fois précisément les moyens grammaticaux de la langue grecque insistant à répéter la pensée par la même forme verbale quoiqu'elle ne s'insère pas dans le système slave. Mentionnons encore le lexique qui doit être au niveau du problème religieux qu'on expose en prenant en considération les doctrines chrétiennes fondamentales. N'oublions pas que l'église orthodoxe serbe n'a pas encore reconnu la traduction de l'Évangile de Vuk Stefanovic-Karadžić d'il y a plus d'un siècle, parce qu'il avait employé quelques mots qui sortent du cadre de bon usage et quoique la traduction de Vuk ne s'éloigne pas beaucoup de celles faites après lui! La qualité essentielle de chaque traduction est réduite à sa clarté. Enfin, nous ne devons pas oublier que les peuples européens n'aient reçu la Bible en leurs langues que très tard.

Si la clarté et la compréhension présentent l'exigence essentielle de chaque texte, il est normal que les deux traductions, roumaine et aroumaine dont nous avons discuté, s' accomodent bien à leurs bénéficiaires aussi du point de vue du problème que nous avons exposé en essayant d'expliquer la différence existante.

25 Les anciennes traductions de la Bible en roumain appliquent aussi le passé simple. Voir, par ex., les exemples que nous offre Virgil Candea, Ratiunea dominanta, Cluj-Napoca 1979 quand il parle des traductions effectuées au XVII ${ }^{e}$ siècle. Cfr. en particulier pp. 141-143. N'oublions pas que la langue officielle d'administration et de l'Eglise orthodoxe roumaine était pour des siècles le slavon (c'est-à-dire une rédaction du vieux slave, naturellement, en caractère cyrilliques qui mettait en relief de nombreux traits balkaniques).

Cfr. Alexandru Georgescu, Perfectul simplu în dialectul daco-român (en Studii de gramatica, vol. II, Bucarest, pp. 29-52). - Il faut souligner en même temps que ce qui est valable pour la Moldavie est valable aussi pour la Moldavie Soviétique (ancienne Bessarabie) ou l'on parle aujourd'hui la langue moldave qui n'est pas reconnue par les romanistes occidentaux ayant en vue que la seule différence entre les deux langues soit l'alphabet. Tandis que le roumain a adopté l'alphabet latin dans la deuxième moitié du siécle passé, le moldave a eu recours à l'alphabet cyrillique après la deuxième guerre mondiale, à savoir après l'annexion de ce territoire à l'Union Soviétique ayant adopté en même temps quelques graphèmes spéciaux sous l'influence de l'alphabet russe. 
Nous y avons pourtant limité nos recherches à un style bien déterminé, ce qui veut dire qu'en passant à d'autres styles, la situation peut être changee ou au moins modifiee. $^{27}$

Il serait aussi intéressant de dire quelques mots sur la disparition des formes simples du prétérit et sur leur remplacement par les formes composées. Nous retournons à une theorie selon laquelle, il y a le mundo narrado et le mundo comentado, dont le premier exige le passé simple (et l' imparfait) et l'autre a recours au passé composé (et au présent). ${ }^{28}$ L'auteur de la dite théorie, acceptable en partie, suit ce procès à partir du latin classique en passant après aux langues romanes et germaniques. Il met en relief la différence entre le mundo narrado et le mundo comentado qui reste visible grâce aux adverbes et aux locutions adverbiales aussi bien quand la forme simple du prétérit disparaît. Mais nous savons aussi qu'une catégorie linguistique peut perdre sa nuance primordiale si elle n'est pas appuyée par la forme verbale bien déterminée, c'est-à-dire en restant dans l'absence psychologique de l'interlocuteur. N'oublions non plus que le même auteur affirme que les formes composées sont imposées d'au-dessus et non d'au-dessous (ayant en vue surtout les innovations proclamées par la Révolution française qui ouvre la porte aux formes composées), tandis que le peuple continuait à utiliser le passé simple. ${ }^{29}$ Les langues périphériques de l'Europe et celles des Balkans donnent une confirmation sérieuse à ce point de vue. N'est-il pas possible d'envisager ce problème en intégrité sans tenir compte des procès accomplis dans les langues slaves?!

27 Au lieu de citer un grand nombre d'articles traitant le problème en question auquel nous avons dédié beaucoup de temps, nous ne mentionnons que notre livre Functiile de baz ă ale aoristului sîrbocroat și ale perfectului simplu românesc în lumina limbilor romanice si balcanice, Pancevo 1972.

Nous avons eu dans nos main la traduction espagnole du livre de Harald Weinrich dont le titre original est Tempus. Besprochene und erzählte Welt, Stuttgart 1971. La traduction espagnole porte le titre Estructura y función de los tiempos en el lenguaje, Madrid 1974. En appliquant sa terminologie, l'auteur a modifie un peu la terminologie qu'offrent les autres linguistes comme "l'enonciation historique" et "l'enonciation du discours" ou "le recit historique" et "le rapport".

Le livre de Weinrich donne des exemples de diverses langues romanes et germaniques en les discutant d'une façon minutieuse et subtile soit du point de vue synchronique ou diachronique. D'autre part, les pages consacrées à l'aspect verbal ne nous offrent rien de nouveau, ce qui est comprehensible parce que l'auteur s'occupe du temps et non pas de l'aspect verbal. Nous y renvoyons en premier lieu aux pages traitant les problèmes des temps en français puisque ce sont les pages les plus convaincantes. 
U prvom (kracem) delu svog izlaganja autor ukratko ukazuje na istoriju Arumuna (Cincara) navodeci da se pominju tek od X. veka, dok se prvi zapis na njihovom jeziku javlja tek u XVII veku. Ističuci da je reč o romanizovanim starosedeocima Balkana, on navodi da je reč o stanovništvu podeljenom danas izmedju Grcke, Albanije, Jugoslavije i Bugarske, a dobrim delom po završetku prvog svetskog rata preseljenom u Rumuniju. Isticući da su Arumeni dali značajan prilog formiranju i kulturi svih balkanskih naroda, autor pominje njihove zahteve, ispoljene naroxito poslednjih decenija, da svoj jezik i svoju kulturu odvoje od rumunskog jezika i rumunske kulture, sto su posebno naglasili odvajajuci svoj alfabet od rumunskog, tj. unoseći posebne foneme uz pozivanje na Gramatiku arumunskogjezika koju je još 1813. godine objavio u Becu M. Bojadži, sastavljenu latiniðnim pismom, pri そ̌emu su objašnjenja data na grčkom i nemačkom jeziku. Ovo delo, koje je doživelo još tri izdanja, 1857. i 1915 godine u Bukurestu i 1988. godine u Frajburgu, predstavlja u isti mah i prvu gramatiku jednog balkanskog jezika.

Ako je do danas arumunski predstavljao jedan od istorijskih dijalekata rumunskog jezika (pored dako-rumunskog, megleno-rumunskog $\mathrm{i}$ istro-rumunskog; ovde ne ubrajamo tzv. moldavski, priznat u SSSR-u, ali ne i van njega), Arumuni smatrajo da danas imaju prava na svoj jezik, koji je već više od hiljadu godina potpuno odeljen od (dako)-rumunskog, što se odražava, pored narodnog blaga, i u nizu novih Casopisa posvećenih umetničkom $\mathrm{i}$ književnom stvaralaštvu, naročito u poeziji.

U drugom (dosta šrem) delu autor se zaustavlja na načinu iskazivanja prošlosti u jeziku Biblije na rumunskom $\mathrm{i}$ arumunskom. Prateći proces zamenjivanja sintetixnih oblika analitižnim u oblasti konjugacije na Sirokim evropskim prostorima, prvenstveno romanskim, ali i germanskim i slovenskim, pa i nekim drugim, autor tvrdi da je do inovacije dołlo u Zapadnoj, Srednjoj i Istočnoj Evropi, dok procesi koji su otuda zračili nisu jos uvek stigli da zahvate i evropsku periferiju. To je i razlog što se u biblijskom stilu današnjeg rumunskog jezika prošlost iskazuje (ako izuzmemo imperfekt) isključivo složenim perfektom, dok je arumunskom, pored imperfekta, $u$ istom stilu redovan oblik jedino aorist (kakav se upotrebljavao nekada i u rumunskom biblijskom stilu), a složeni oblik je rezervisan isključivo za dijalog. 